\title{
Some constructive roads to Tychonoff
}

\author{
Steven Vickers \\ School of Computer Science, University of Birmingham, \\ Birmingham, B15 2TT, UK. \\ s.j.vickers@cs.bham.ac.uk
}

CLARENDON PRESS • OXFORD

2004 


\begin{abstract}
The Tychonoff Theorem is discussed with respect to point-free topology, from the point of view of both topos-valid and predicative mathematics.

A new proof is given of the infinitary Tychonoff Theorem using predicative, choice-free methods for possibly undecidable index set. It yields a complete description of the finite basic covers of the product.
\end{abstract}

\title{
0.1 Introduction
}

The Tychonoff theorem says that a product of compact topological spaces is still compact. (I do not assume Hausdorff separation here. By "compact" I mean having the finite subcover property, as in the Heine-Borel theorem; Bourbaki calls this "quasicompact".) For finitary products this is fairly elementary. Surprisingly, the result extends to infinitary products, but there is a price - the axiom of choice has to be assumed. In fact, as is well known, the infinitary Tychonoff theorem is equivalent to the axiom of choice [Kel50].

This is sometimes offered as a reason for using the axiom of choice: with it, one can prove many genuinely useful results such as infinitary Tychonoff. There are more that don't rely on the full power of the axiom of choice, but still need classical principles. These include the Heine-Borel theorem (that any bounded closed interval in the real line is compact) [FG82] and the Hofmann-Mislove theorem (that for a sober space there is a bijection between compact saturated subspaces and Scott open filters in the topology) [HM81], [Vic89]. The suggestion is that the theory of topology is unavoidably impoverished if it cannot call on all the classical reasoning principles.

It therefore comes as a surprise to discover that in point-free topology, such theorems can often be proved constructively, at least if stated correctly. In this paper we illustrate this with the Tychonoff theorem. Its validity is quite unequivocal, with no special assumptions. For instance, we do not have to assume excluded middle either, and the indexing set does not have to have decidable equality. Neither do we have to use impredicative constructions. Tychonoff is actually a robust part of constructive topology.

The problem lies not in forsaking choice, but in insisting on a point-set formulation of topology. Three things are jointly incompatible: Tychonoff, constructivity, and point-set topology. If we wish to keep Tychonoff then we must drop one of the others. However, it does not have to be the constructivity. The aim of this paper is to describe how it works if we decide to drop the point-set formulation.

\subsubsection{Point-free topology}

In the usual point-set topology, a topological space is a set equipped with additional topological structure that can be axiomatized in various ways, classically equivalent to each other. Our preferred form here is via the open sets, defining a topology to be a family of subsets of the set of points, closed under finite intersection and arbitrary union.

By contrast I shall use the phrase "point-free topology" as a generic label for approaches that do not start by assuming a set of points of the space. In these 
approaches, a topological space cannot in general be described as a set of points equipped with extra structure.

In practice, points are not excluded from the "point-free" discussion. Normally they are at least helpful for keeping in touch with topological intuitions, and there are tricks of categorical logic by which rigorous arguments can be conducted in terms of points. However, we cannot assume that the totality of all points can be collected together as a set. A space is something more general than a set. What is more, when a set of points can be extracted, we cannot assume that it adequately represents the collection of all points.

There are two main versions of point-free topology that I shall consider, and they are genuinely different. They amount to the elaboration of point-free ideas in two radically different foundational settings, namely topos theory and predicative type theory. (I know more about the topos theory side, so you must excuse me if sometimes my knowledge of type theory is deficient.)

The first version, used in topos theory, is locales [Joh82]. I shall explain those first because in many ways it helps to clarify what the second version is achieving. The second version, used in predicative type theory, is formal topology [Sam87].

\subsubsection{Locales}

Locale theory is based on the most direct interpretation of the phrase "pointfree topology". In point-set topology, the topology is the collection of open sets. The idea is to use this in a point-free way by treating it as an abstract lattice, forgetting that it was ever a set of subsets of some set of points.

The standard introduction is [Joh82] (or see also [Vic89]), and we give just a brief overview.

Definition 0.1 A frame is a complete lattice in which binary meet distributes over arbitrary join.

A frame homomorphism is a function between frames that preserves arbitrary joins and finite meets.

(Naturally, the arbitrary joins and finite meets here correspond to the arbitrary unions and finite intersections of open sets.)

We write $\mathbf{F r}$ for the category of frames and frame homomorphisms, and Loc for its opposite - the category of locales and (continuous) maps. By this definition a locale "is" just a frame, but it is best to keep them notationally distinct since the language for locales is quite different from that for frames. For instance, products of locales are coproducts of frames; sublocales are quotient frames. Locales are frames "pretending to be" topological spaces, and it is best to keep up the pretence. If $X$ is a locale we shall write $\Omega X$ for the corresponding frame; and if $f: X \rightarrow Y$ then we write $f^{*}: \Omega Y \rightarrow \Omega X$ for the corresponding frame homomorphism.

Locales, then, are the spaces in this version of point-free topology.

The points of a space $X$ should be the maps from the one-point space 1 to $X$, and we can implement this with locales. The (discrete) topology on 1 is 
the powerset $\mathcal{P} 1$. In the internal language of toposes $\mathcal{P} 1$ is just the subobject classifier $\Omega$, and we shall write it as such. The best way to think of $\Omega$ is as the "set of truthvalues", which classically is $\{$ true, false $\}$. A point $x$ of a locale $X$ is then a frame homomorphism $x^{*}: \Omega X \rightarrow \Omega$. Now any function to $\Omega$ is equivalently described by its true kernel, the inverse image of $\{$ true $\}$, and the function $x^{*}$ is a frame homomorphism iff its true kernel is a completely prime filter, an upper set that is closed under finite meets and inaccessible by arbitrary joins. (Note that some of the other common characterizations of point ( [Joh82], [Vic89]) are not constructively equivalent. Some of this is because in the absence of excluded middle the homomorphism $x^{*}$ is not determined by its false kernel $\left(x^{*}\right)^{-1}$ \{false $\}$.)

In topos-valid mathematics, we can construct the set $\operatorname{pt}(X)$ of points of $X$. However, as we shall see it may be defective. Even classically there may fail to be enough points to distinguish between the opens, so that $\Omega X$ does not embed in $\mathcal{P} \operatorname{pt}(X)$.

There are two mismatches between locales and topological spaces.

The first is that locales are intrinsically sober. This just means that the space contains all the points that can be reconstructed from the topology, and so must be a feature of any point-free approach. A non-sober topological space may have distinct elements of the point-set that cannot be distinguished topologically because they are in the same opens. In other words, the space may fail to be $T_{0}$. A non-sober space may also lack points such as the directed joins (with respect to the specialization order) present in every sober space. For example, a poset with its Alexandrov topology (the opens are the upper sets) is not sober in general, and to make it so you have to add directed joins by going to the ideal completion.

That first mismatch is perhaps not so serious. One can argue that all decent spaces should be sober. Alternatively, you can express the duplications and omissions of points in a non-sober space by a map from a discrete locale $X$ $(\Omega X=\mathcal{P} X)$ to another locale. (These are the topological systems of [Vic89].)

The second mismatch is more fundamental, and that is that locales do not always have enough points - they may fail to be spatial. Indeed, some non-trivial locales fail to have any points at all. The technical manifestation of this is that the frame homomorphism $\Omega X \rightarrow \mathcal{P} \operatorname{pt}(X)$ fails to be $1-1$. In classical mathematics the non-spatial locales are generally pathological, since the axiom of choice can be used to show the existence of enough points for wide classes of useful locales. Constructively, however, even necessary locales need not be spatial. A good example is the real line. The localic real line for which good mathematics holds - for instance, the Heine-Borel theorem - is the one presented as $L(\mathbb{R})$ in [Joh82, IV.1.1]. Constructively this can easily be non-spatial [FH79].

Such non-spatiality may seem pathological wherever it occurs. After all, what kind of topological structure can it be that is not supported by the points? However, the well-known topological theorems work better with the non-spatial locales - the purpose of this paper is to illustrate this for Tychonoff, and HeineBorel has also been mentioned. Here's one way to imagine how the points might 
not be the whole story. Often we are interested in other pieces within the space, for example line elements (maps from $[0,1]$ ). Say a generalized point of $X$ is a map from some domain $Y$ (the "stage of definition") to $X$. Even if there are insufficient "global" points (stage of definition is 1), there are still plenty of generalized points. In fact, this comes rather cheaply, since the generic point (the identity map from $X$ to itself) is enough for most purposes.

It was understood quite early (see e.g. [JT84]) that locale theory is constructive in the topos-valid sense. The notion of internal frame can be defined in any topos, and the constructions one needs (for example, coproduct of frames for product of locales) can be carried through. Moreover, there is an extremely important relativization principle. Suppose $X$ is a locale. There is a topos associated with it, namely its topos of sheaves which I shall write $\mathcal{S} X$. What are the internal frames in $\mathcal{S} X$ ? It turns out they are equivalent to continuous maps (of locales) with codomain $X$. Hence a constructive result about locales, interpreted in $\mathcal{S} X$, can be turned into a result about maps into $X$-in other words, generalized points of $X$. Thus the topos-valid constructivist discipline delivers a payoff. It is not merely a claim to moral superiority.

One benefit is that constructive arguments about points can be applied also to the generalized points (as "points at another stage of definition"). The sufficiency of these can therefore validate spatial reasoning about point-free topologies. This is exploited in [Vic99], [Vic04a], which also explain why the more stringent geometric constructivism is needed to ensure that the arguments can be transferred from one stage of definition to another.

Topos-valid constructivism is completely choice-free. In general it is not even possible to choose one element out of two. Consider for example the topos of sheaves over the circle $O$. If the circle is represented as the complex numbers of unit modulus, then the squaring function $z \mapsto z^{2}$, the Möbius double cover of the circle, is a local homeomorphism and hence equivalent to a sheaf. In the internal language of the topos $\mathcal{S O}$, it is a set $X$, finite with decidable equality, satisfying

$$
\exists x, y \in X .(x \neq y \wedge \forall z \in X .(z=x \vee z=y))
$$

but with no element $1 \rightarrow X$.

\subsubsection{Formal topologies}

Despite the success of locales in topos-valid mathematics, its use of impredicative constructions troubles some constructivists. These are constructions that presuppose a collection that already includes what one is trying to construct. The question often arises in connection with powersets $\mathcal{P} X$, since if one is trying to construct some subset of $X$ it would be impredicative to presuppose that $\mathcal{P} X$ - the set of all subsets including the one being constructed - is already to hand. In general predicative mathematics would not admit $\mathcal{P} X$ as a set.

Unfortunately, many of the constructions of locale theory are impredicative. This includes the construction of $\mathrm{pt}(X)$, though I have already argued that we 
may be able to do without it. More seriously, however, the frames themselves are impredicative. This is most obvious for discrete locales, whose frames are powersets. Then other frames such as $\Omega L(\mathbb{R})$ mentioned above, require impredicative constructions. And the very definition of frame, as complete lattice, describes joins in $A$ by a function from $\mathcal{P} A$ to $A$.

One way to understand formal topology is that it is obliged to do "locale theory without the frames". In fact, techniques for this are already present in the ordinary practice of locale theory.

The impredicative step is normally in ensuring that all joins (of subsets) are present. But it is often enough to work with a base of the topology, so that every open is a join of basic opens, and the base can often be constructed predicatively. More generally one might use a subbase, so that every open is a join of finite meets of subbasics. However, this makes no difference to the predicativity, since the finite powerset construction is inductive. (As elsewhere in this paper, "finite" means Kuratowski finite. The finite powerset $\mathcal{F} X$ can also be represented algebraically as the free semilattice over $X$.)

In locale theory this use of bases or subbases appears in algebraic form, as presenting a frame by generators and relations. In [Joh82] it underlies the construction of $\Omega L(\mathbb{R})$. This is generated by basics $(p, q)(p \in \mathbb{Q} \cup\{-\infty\}, q \in$ $\mathbb{Q} \cup\{\infty\})$ subject to relations-

$$
\begin{aligned}
1 & =(-\infty, \infty) \\
(p, q) \wedge\left(p^{\prime}, q^{\prime}\right) & =\left(\max \left(p, p^{\prime}\right), \min \left(q, q^{\prime}\right)\right) \\
(p, q) & \leq 0 \text { if } p \geq q \\
(p, s) & \leq(p, r) \vee(q, s) \text { if } p \leq q<r \leq s \\
(p, q) & \leq \bigvee\left\{\left(p^{\prime}, q^{\prime}\right) \mid p<p^{\prime}<q^{\prime}<q\right\} \text { if } p<q
\end{aligned}
$$

The presentation itself is predicative: the set of generators, the set of relations and the sets of disjuncts in infinitary joins are all constructed predicatively. (This idea is explored in great detail in [Vic04a].) Hence, within predicative mathematics, the presentation can be used as a surrogate for the frame. This, roughly speaking, is what a formal topology is. More precisely, this is an inductively generated formal topology.

One sees many different definitions in formal topology. To give some shape to the issues involved, I mention three different modes of variation.

1. There are different kinds of structure that can be interpreted as generators and relations. The different forms of structure tend to come out as different definitions of formal topology. For example, the site as described in [Joh82, II.2.11] provides one particular form of generators and relations. The generators are required to form a meet semilattice, and there are implied relations to say that the semilattice meet is preserved in the frame.

2. It used to be customary in formal topology to require spaces to be open in the sense of [JT84], namely that the unique map to 1 should be an open map. (Classically, all locales are open. But constructively it becomes an important 
issue.) For this a positivity predicate is needed on the basics in order to say in a positive way which are non-empty [Joh84], [Neg02]. A formal topology without positivity predicate is often called a formal cover.

3. The original definitions of formal topology required a specification of the full cover relation $\triangleleft$, i.e. to say for each set $U$ of generators which generators $a$ were to be less than $\bigvee U$. Of course, $\triangleleft$ is not itself a predicative set. But the information amounts to describing how proofs of $a \triangleleft U$ may be constructed. More recently [CSSV03] showed how to use an axiom set, effectively a set of relations, to generate the full cover relation $\triangleleft$. Such a structure is called an inductively generated formal cover. Not all formal covers can be inductively generated.

In what follows, we shall use the following definition of inductively generated formal cover.

Definition 0.2 A flat site is a structure $\left(P, \leq, \triangleleft_{0}\right)$ where $(P, \leq)$ is a preorder (i.e. transitive and reflexive), and $\triangleleft_{0} \subseteq P \times \mathcal{P} P$ has the following flat stability property: if $a \triangleleft_{0} U$ and $b \leq a$, then there is some $V \subseteq b \downarrow U$ such that $b \triangleleft_{0} V$.

(For subsets or elements $U$ and $V$, we write $\downarrow U$ for the down closure of $U$ with respect to $\leq$ and $U \downarrow V$ for $\downarrow U \cap \downarrow V$.)

The reason for calling this "flat" is as follows. In category theory there is a notion of flat functor from $C$ to Set such if $C$ is cartesian (has all finite limits) then flatness is equivalent to the functor being cartesian (preserves finite limits). See, e.g., [MLM92]. (This is also related to the notion of flat module in ring theory, using the idea from enriched category theory that a functor from $C$ to Set can be considered a kind of module over $C$.) The notion of ordinary site [Joh82] is essentially a special case of our flat site in which $P$ is a meet semilattice, i.e. a cartesian poset. In categorical logic, points of the corresponding locale can be understood as certain cartesian functors from $P$ to Set, and in the flat site these generalize to the flat functors from $P$ to Set.

Definition 0.2 is just a rephrasing of the localized axioms sets of [CSSV03]. Their axiom set is an indexed family $I(a)$ set $[a: P]$ together with a family of subsets $C(a, i) \subseteq P[a: P, i: I(a)]$. "Localized" means that for any $a \leq c$ and $i \in I(c)$, there exists $j \in I(a)$ such that $C(a, j) \subseteq a \downarrow C(c, i)$. Then our $\triangleleft_{0}$ comprises the instances of $a \triangleleft_{0} C(a, i)$.

The full formal cover $\triangleleft$ is generated from this by rules

- $\frac{a \in U}{a \triangleleft U} \quad$ (reflexivity)

- $\frac{a \leq b \quad b \triangleleft U}{a \triangleleft U} \quad(\leq$-left $)$

- $\frac{a \triangleleft_{0} V \quad V \triangleleft U}{a \triangleleft U} \quad$ (infinity)

The flat site gives rise to a frame presentation in which the generators are the elements of $P$, and the relations are: 


$$
\begin{aligned}
1 & \leq \bigvee P \\
a \wedge b & =\bigvee\{c \mid c \leq a, c \leq b\} \\
a & \leq \bigvee U\left(a \triangleleft_{0} U\right)
\end{aligned}
$$

\subsection{Compactness}

The notion of compactness translates easily from spaces to locales. A locale $X$ is compact if the top open $1 \in \Omega X$ has the property that every cover has a finite subcover. Alternatively, if a directed subset $S$ has its join equal to 1, then 1 must already be in $S$-i.e. $\{1\}$ is Scott open.

This is straightforward, but notice that compactness of the locale and its space of points $\operatorname{pt}(X)$ become two unrelated properties. Let $\Omega \operatorname{pt}(X)$ be the topology induced on $\operatorname{pt}(X)$, the image of the frame homomorphism $\Omega X \rightarrow \mathcal{P} \operatorname{pt}(X)$, and let us write $F$ for the filter of $\Omega X$ comprising those opens that map to top in $\Omega \operatorname{pt}(X)$. If $F$ is not spatial, so $\Omega X \rightarrow \Omega \operatorname{pt}(X)$ is not 1-1, then $F$ may be different from $\{1\}$. Compactness for $\operatorname{pt}(X)$ is equivalent to saying that $F$ is $\operatorname{Scott}$ open -if a directed join $\bigvee S$ is in $F$, then $S$ already has an element in $F$. Scott openness of $F$ neither implies nor is implied by Scott openness of $\{1\}$.

In fact, this explains something of the gap between spatial Tychonoff (requiring choice in general) and localic Tychonoff (no choice needed). Even for spatial locales, the product need not be spatial. Hence compactness of the product locale does not imply compactness of the product space - the two questions are separate.

Despite the simplicity of the definition of localic compactness, in practice it is a non-trivial question. This is because it is rare for the frame structure to be given explicitly in a concrete form. For instance, if the frame is presented by basic generators and relations it is not in general clear when one open is covered by a family of basics. The impredicative definition of the cover relation - in effect "the least frame congruence containing the relations" - is little help. In particular this is a problem with a product $\prod_{i} X_{i}$ of locales, whose coproduct frame is most easily presented by a "disjoint union" of presentations for the frames $\Omega X_{i}$. This coproduct frame may also be described as a tensor product of complete join semilattices, but that is no real help here because - just as with linear tensor products - the elements cannot be expressed in any canonical form.

A direct predicative approach requires some knowledge of the full cover relation $\triangleleft$.

Let us outline some sharper approaches to the question.

\subsubsection{Preframes}

A preframe is a poset with finite meets and directed joins, with meet distributing over directed joins. A preframe homomorphism preserves finite meets and directed joins.

The importance of preframes lies in the fact that for a subset $F$ of a frame $\Omega X, F$ is a Scott open filter iff its characteristic function to $\Omega$ is a preframe 
homomorphism - filteredness and Scott openness correspond to preservation of finite meets and directed joins respectively. A simple proof of Tychonoff using preframe techniques was given in [JV91].

This can be expressed neatly within locale theory by the upper powerlocale $P_{U} X$ (see [Vic97], and also [Vic04a]). By definition its frame $\Omega P_{U}(X)$ is generated as frame by the elements of $\Omega X$, respecting the preframe structure of $\Omega X$. Hence maps from $Y$ to $P_{U}(X)$ are equivalent to preframe homomorphisms from $\Omega X$ to $\Omega Y$ and the points of $P_{U} X$ are the Scott open filters of $\Omega X$.

Johnstone's localic version of the Hofmann-Mislove Theorem - see [Vic97], deriving from [Joh85] - says that the Scott open filters of $\Omega X$ correspond to compact fitted sublocales of $X$, where a sublocale is fitted if it is a meet of open sublocales. (Classically this corresponds to subspaces that are saturated, i.e. upper closed under the specialization order.) Hence $P_{U} X$ is indeed a powerlocale, its points being certain sublocales of $X$. The correspondence is order reversing, and a bottom point of $P_{U} X$ corresponds to the greatest possible compact sublocale of $X$, namely $X$ itself.

[Vic95] shows that proving compactness of $X$ is equivalent to finding a bottom point $\perp$ of $P_{U}(X)$, in the strong sense that the composite !; $\perp: P_{U} X \rightarrow$ $1 \rightarrow P_{U} X$ is less than the identity map in the specialization order. This condition says that $\perp$ is not just least amongst the global points $1 \rightarrow P_{U} X$. It is also less than the generic point id : $P_{U} X \rightarrow P_{U} X$, and this makes it least amongst all generalized points $Y \rightarrow P_{U} X$.

All this is impredicative, but it can be made predicative. The "preframe coverage theorem" of [JV91] shows how to convert presentations of frames by generators and relations into preframe presentations of the same frames, and so shows how to convert frame presentations of $\Omega X$ into frame presentations of $\Omega P_{U} X$. This can be made into a predicative construction within formal topology.

Proposition 0.3 Let $\left(P, \leq, \triangleleft_{0}\right)$ be a flat site presenting locale $X$. Then $P_{U} X$ is presented by the flat site $\left(\mathcal{F} P, \sqsubseteq_{L}, \triangleleft_{0}\right)$ where $\mathcal{F} P$ is the (Kuratowski) finite powerset of $P$, ${ }_{L}$ is the lower order on $\mathcal{F} P$, defined by $S \sqsubseteq_{L} T$ iff for every $x \in S$ there is some $y \in T$ with $x \leq y$, and $\triangleleft_{0}$ is given by the following.

Suppose $a_{i} \triangleleft_{0} U_{i}(1 \leq i \leq n)$. Let $A=\left\{a_{i} \mid 1 \leq i \leq n\right\}$. Then for every $S \in \mathcal{F} P$,

$$
A \cup S \triangleleft_{0}\left\{T \cup S \mid T \sqsubseteq_{L} \bigcup_{i=1}^{n} U_{i}\right\} .
$$

Proof We merely sketch the proof here. $\Omega X$ is presented as frame by generators $P$ and relations as given after Definition 0.2. We can write it as

$$
\begin{aligned}
\operatorname{Fr}\langle P \text { (qua preorder) }| & \leq \bigvee P \\
a \wedge b & \leq \bigvee(a \downarrow b) \\
a & \left.\leq \bigvee U \quad\left(\text { if } a \triangleleft_{0} U\right)\right\rangle
\end{aligned}
$$


where "qua preorder" indicates extra implicit relations to say the preorder structure of $P$ is preserved in the frame. The free join semilattice over the preorder $P$ is $\mathcal{F} P / \sqsubseteq_{L}$, with join represented by union. We can therefore transform the presentation into an equivalent one,

$$
\begin{aligned}
\operatorname{Fr}\left\langle\mathcal{F} P / \sqsubseteq_{L} \quad \text { (qua } \vee \text {-semilattice) }\right| 1 & \leq \bigvee^{\uparrow} \mathcal{F} P \\
(\{a\} \cup S) \wedge(\{b\} \cup S) & \leq \bigvee^{\uparrow}\left\{(T \cup S) \mid T \sqsubseteq_{L}\{a\}, T \sqsubseteq_{L}\{b\}\right\} \\
(\{a\} \cup S) & \left.\leq \bigvee^{\uparrow}\left\{(T \cup S) \mid T \sqsubseteq_{L} U\right\} \quad\left(\text { if } a \triangleleft_{0} U, S \in \mathcal{F} P\right)\right\rangle .
\end{aligned}
$$

$\left(\mathrm{V}^{\uparrow}\right.$ indicates a join that is known to be directed. We have not distinguished between on the one hand the elements of $\mathcal{F} P / \sqsubseteq_{L}$, equivalence classes with respect to the equivalence relation corresponding to $\sqsubseteq_{L}$, and on the other hand the elements of $\mathcal{F} P$ that represent them.)

Here the relations have been put in the join stable form required for the preframe coverage theorem [JV91], and from that we find that $\Omega P_{U} X$ can be given exactly the same presentation, except that "qua $\vee$-semilattice" is replaced by "qua preorder under $\sqsubseteq_{L}$ ".

Using some induction on the finite sets, we find that the middle relation scheme is equivalent to

$$
(A) \wedge(B) \leq \bigvee^{\uparrow}\left\{(T) \mid T \sqsubseteq_{L} A, T \sqsubseteq_{L} B\right\}
$$

for $A, B \in \mathcal{F} P$. Thus those first two relation schemes are equivalent to the implicit relations in a flat site on $\left(\mathcal{F} P, \sqsubseteq_{L}\right)$. The final relation scheme does not satisfy the flat stability condition, but it is equivalent to the relation scheme given in the statement of the theorem, which does.

It follows that the upper powerlocale can also be accessed in predicative theories. The same compactness criterion - existence of a suitable point of the powerlocale - can then be expressed. The argument given so far for its correctness has used the impredicative results about preframes, but [Vic02] gives a direct predicative proof. After a little simplification, it appears there as

Theorem 0.4 Let $\left(P, \leq, \triangleleft_{0}\right)$ be a flat site presenting a locale $X$. Then $X$ is compact iff there is a subset $F$ of $\mathcal{F} P$ such that-

1. $F$ is upper closed with respect to $\sqsubseteq_{L}$.

2. $F$ is inhabited.

3. If $a \triangleleft_{0} U$ and $\{a\} \cup T \in F$, then $U_{0} \cup T \in F$ for some $U_{0} \in \mathcal{F} U$.

4. If $S \in F$ then $P \triangleleft S$ (i.e. $\forall g \in P . g \triangleleft S$ ).

In that case, $F$ is necessarily the set of all finite basic covers of $X$.

We note briefly that it is not only preframe homomorphisms that can be captured predicatively in this way. So too can arbitrary dcpo morphisms (Scott 
continuous functions) between frames. (A dcpo is a directed complete poset, i.e. a poset with all directed joins.) This is done using the double powerlocale $\mathbb{P} X$ ( [JV91], [Vic04a]), for which the frame $\Omega \mathbb{P} X$ is the free frame generated by $\Omega X$ and preserving its dcpo structure. The maps from $Y$ to $\mathbb{P} X$ are equivalent to Scott continuous functions from $\Omega X$ to $\Omega Y$, and $\mathbb{P} X$ can be constructed by predicative constructions on presentations ( [Vic04a], [VT04]). Thus the double powerlocale can also be defined on inductively generated formal topologies.

\subsection{Tychonoff}

We can now illustrate the techniques with a proof of Tychonoff that is valid both in topos theory and in predicative mathematics. It assumes neither finiteness nor decidability of equality for the indexing set for the locales of which the product is taken.

In topos-valid locale theory this result appears to be due to Vermeulen [Ver86]. In formal topology, following an earlier treatment of [Coq92], the infinitary Tychonoff was proved in [NV97] without choice but under the assumption that the indexing set had decidable equality. This arose from the way that basic opens for the product $\prod_{i} X_{i}$ - finite meets of opens taken from the componentswere normalized into elements of $\prod_{i} \Omega X_{i}$ in which all but finitely many components are 1 . This normalization can only be done effectively if there is decidable equality for indexes.

Subsequently, [Coq03] gave a simple choice-free predicative proof without decidable equality. His argument rests on the fact that for any spectral locale $X$, there is a least compact sublocale $Y$ whose fitted hull (= saturation) is the whole of $X$. It follows that every sublocale between $Y$ and $X$ is compact. Coquand shows how to describe a product locale in this way. (Coquand has remarked separately that the underlying construction is a localic version of the "maximal spectrum" described spatially in [Joh82, II.3.5].)

Though elegant, Coquand's proof requires some preparation before it can be put into effect. It relies on having each locale presented using a distributive lattice of generators for which the order coincides with the order in the presented frame, and getting that is non-trivial. We now give a proof that shows how from general flat sites, the finite covers of the product can be calculated.

Proposition 0.5 Let $\left(P_{i}, \leq, \triangleleft_{0}\right)$ be a flat site for each $i \in I$. Then the product of the corresponding locales is presented by a flat site $\left(P, \leq, \triangleleft_{0}\right)$ defined as follows.

First, let $\left(P^{\prime}, \leq\right)=\sum_{i \in I} P_{i}$ be the poset coproduct. As a set it is the disjoint union, $\left\{(i, x) \mid i \in I, x \in P_{i}\right\}$, with $(i, x) \leq(j, y)$ iff $i=j$ and $x \leq y$ in $P_{i}$. Its elements are subbasics.

Now define $P=\mathcal{F} P^{\prime}$ ordered by $\sqsubseteq_{U}$, i.e. $A \leq B$ iff $\forall b \in B . \exists a \in A . a \leq b$. Its elements represent finite meets of subbasics. $P / \sqsubseteq_{U}$ is in fact the free meet semilattice over the poset $P^{\prime}$, meet being represented by union.

Covers are defined as follows:

1. If $i \in I$ and $B \in P$, then 


$$
B \triangleleft_{0}\left\{\{(i, a)\} \cup B \mid a \in P_{i}\right\} .
$$

2. If $i \in I, a, a^{\prime} \in P_{i}$ and $B \in P$, then

$$
\left\{(i, a),\left(i, a^{\prime}\right)\right\} \cup B \triangleleft_{0}\left\{\{(i, c)\} \cup B \mid c \leq a, c \leq a^{\prime}\right\} .
$$

3. If $i \in I, a \triangleleft_{0} U$ in $P_{i}$, and $B \in P$, then

$$
\{(i, a)\} \cup B \triangleleft_{0}\{\{(i, u)\} \cup B \mid u \in U\} .
$$

Proof First note that this is indeed a flat site; in fact it is an ordinary site. ( $P$ is a meet semilattice and the coverage has meet stability.)

The frame for the product is presented by putting together the presentations for the original frames. For clarity, let us write $\alpha_{i}$ for the injections of generators. Then the frame is presented as-

$$
\begin{aligned}
\operatorname{Fr}\left\langle\alpha_{i}(a)\left(i \in I, a \in P_{i}\right)\right| & \\
\alpha_{i}(a) & \leq \alpha_{i}\left(a^{\prime}\right) \quad\left(i \in I, a \leq a^{\prime} \text { in } P_{i}\right) \\
1 & \leq \bigvee_{a \in P_{i}} \alpha_{i}(a) \quad(i \in I) \\
\alpha_{i}(a) \wedge \alpha_{i}\left(a^{\prime}\right) & \leq \bigvee\left\{\alpha_{i}(c) \mid c \leq a, c \leq a^{\prime}\right\} \quad\left(i \in I, a, a^{\prime} \in P_{i}\right) \\
\alpha_{i}(a) & \left.\leq \bigvee_{u \in U} \alpha_{i}(u) \quad\left(i \in I, a \triangleleft_{0} U \text { in } P_{i}\right)\right\rangle .
\end{aligned}
$$

This is isomorphic to

$$
\begin{aligned}
\mathbf{F r}\langle P(\text { qua } & \wedge \\
B & \leq \cup \text { semilattice }) \mid \\
\left\{(i, a),\left(i, a^{\prime}\right)\right\} \cup B & \leq \bigvee\left\{\{(i, c)\} \cup B \mid c \leq a, c \leq a^{\prime}\right\} \quad\left(i \in I, a, a^{\prime} \in P_{i}, B \in P\right) \\
\{(i, a)\} \cup B & \left.\leq \bigvee_{u \in U}\{(i, u)\} \cup B \quad\left(i \in I, a \triangleleft_{0} U \text { in } P_{i}, B \in P\right)\right\rangle .
\end{aligned}
$$

The "qua" notation denotes additional relations to preserve the $\wedge$-semillatice structure (concretely $\cup$ ) of $P$.

In one direction, the isomorphism takes $\alpha_{i}(a) \mapsto(\{(i, a)\})$, while in the other it takes $A \longmapsto \bigwedge\left\{\alpha_{i}(a) \mid(i, a) \in A\right\}$.

To say this predicatively, we are describing two mutually inverse continuous maps between the corresponding formal topologies.

This second presentation corresponds to the product site described in the statement.

To prove Tychonoff, we use Theorem 0.4. Suppose we have flat sites $\left(P_{i}, \leq\right.$ ,$\left.\triangleleft_{0}\right)$ and are given sets $F_{i}$ describing compactness for the $P_{i}$ s. We show how to construct a corresponding set $F$ for the product. The main point of interest is that $F$ itself can be defined without reference to the full coverage $\triangleleft$. The full coverage and its inductive generation need to be considered only when showing that every set in $F$ covers the product space; but this is hardly surprising, because the corresponding facts for the $F_{i}$ s were described in terms of $\triangleleft$. 
We must find a way to characterize the finite basic covers. We give an informal argument as motivation; applying the theorem will confirm its correctness.

Each subbasic $(i, a)$ in $P^{\prime}$ is of the form (spatially) $\left\{\left(x_{j}\right)_{j \in I} \mid x_{i} \in a\right\}$ : think of this as a product of $a$ (at $i) \times P_{j}$ (everywhere else). A basic in $P$ is a set of these representing a meet, and that can be thought of as a product of specified $a$ 's at finitely many specified $i$ 's, times $P_{j}$ everywhere else. (However, we must also allow for the fact that some $i$ may occur more than once.)

We want to know when a join of these meets covers the entire product, and the trick is to use distributivity to change it to a meet of joins. Then every one of the joins must be the whole product.

By distributivity,

$$
\bigvee_{A \in \mathcal{A}} \wedge A=\bigwedge_{\gamma \in \operatorname{Ch}(\mathcal{A})} \bigvee \operatorname{Im} \gamma
$$

where $\operatorname{Ch}(\mathcal{A})$ is the set of choices of $\mathcal{A}$, i.e. [Vic04b] the finite total relations $\gamma$ from $\mathcal{A}$ to $\bigcup \mathcal{A}$ such that if $(A, a) \in \gamma$ then $a \in A$, and $\operatorname{Im} \gamma$ is the image of $\gamma$ (under the second projection from $\mathcal{A} \times \cup \mathcal{A}$ to $\bigcup \mathcal{A}$ ).

Now consider a finite join of subbasics $\bigvee B$. This is (it will turn out) a cover of the entire product iff at some $i$ its components cover $P_{i}$. Classically one sees this as follows. Suppose at every $i$ we have some point $x_{i}$ that is not in any subbasic $b$ in $B$. Then the point $\left(x_{i}\right)_{i \in I}$ is not in $\bigvee B$. Hence (classically) if $\bigvee B$ does cover the product, then there is some $i$ and some finite cover $S$ of $P_{i}$ (so $\left.S \in F_{i}\right)$ such that $\{i\} \times S \subseteq B$.

This idea lies behind our definition of $F$ in the Theorem.

Theorem 0.6. (Infinitary Tychonoff) Let $\left(P_{i}, \leq, \triangleleft_{0}\right)(i \in I)$ be flat sites for compact spaces, equipped with sets $F_{i} \subseteq \mathcal{F} P_{i}$ satisfying the conditions of Theorem 0.4. Let the product site $P$ be defined as above.

Let $F \subseteq \mathcal{F} P$ be defined such that $\mathcal{A} \in F$ iff for every $\gamma \in \operatorname{Ch}(\mathcal{A})$ there is some $i$ and some $S \in F_{i}$ such that for every $a \in S$ we have $(i, a) \in \operatorname{Im}(\gamma)$.

Then $F$ satisfies the conditions of Theorem 0.4 for $P$, and hence shows that $P$ is compact.

Proof In the definition of $F$, we should like to say that for some $i, \operatorname{Im} \gamma$ covers $P_{i}$ : or $\{a \mid(i, a) \in \operatorname{Im} \gamma\} \in F_{i}$. But we have to be somewhat careful, since if $I$ does not have decidable equality then $\{a \mid(i, a) \in \operatorname{Im} \gamma\}$ need not be finite. Nonetheless, let us abuse language and say "Im $\gamma$ covers $P_{i}$ ". Note also that if some $F_{i}$ contains $\emptyset$, so that $P_{i}$ gives an empty locale and so does the whole product, then every $\mathcal{A}$ is in $F$.

We verify the four conditions in Theorem 0.4 .

Condition $1, F$ is upper closed with respect to $\sqsubseteq_{L}$. Suppose $\mathcal{A} \in F$ and $\mathcal{A} \sqsubseteq_{L} \mathcal{B}$. Let $\delta \in \operatorname{Ch}(\mathcal{B})$. If $A \in \mathcal{A}$ then $A \sqsubseteq_{U} B$ for some $B \in \mathcal{B}$. There is some $b \in B \cap \operatorname{Im} \delta$, and $a \leq b$ for some $a \in A$. In short, $\forall A \in \mathcal{A}$. $\exists a \in A$. $\exists b \in \operatorname{Im} \delta . a \leq$ $b$. It follows that there is some $\gamma \in \operatorname{Ch}(\mathcal{A})$ such that $\operatorname{Im} \gamma \sqsubseteq L \operatorname{Im} \delta$. Now because $\mathcal{A} \in F$ we deduce that $\operatorname{Im} \gamma$ covers some $P_{i}$, and it follows that $\operatorname{Im} \delta$ covers the same $P_{i}$ so $\mathcal{B} \in F$. 
Condition 2, $F$ is inhabited. $\{\emptyset\}$ is vacuously in $F$, because it has no choices.

Condition 3. There are three parts to check, corresponding to the three axiom schemes in Proposition 0.5. We use Lemma 0.7, which is proved separately.

For scheme 1 , we need that if $i \in I, B \in P$ and $\{B\} \cup \mathcal{C} \in F$, then there is some $S^{\prime} \in \mathcal{F} P_{i}$ such that $\left\{\{(i, a)\} \cup B \mid a \in S^{\prime}\right\} \cup \mathcal{C} \in F$. In the lemma, take $S=\emptyset$ and $\phi$ the whole of $P_{i}$. Given $T$, there is some $S^{\prime}$ such that $S^{\prime} \cup T$ covers $P_{i}$; just choose $S^{\prime}$ to be any element of $F_{i}$ (which is inhabited by hypothesis).

For scheme 2, if $i \in I, a_{1}, a_{2} \in P_{i}, B \in P$ and $\left\{\left\{\left(i, a_{1}\right),\left(i, a_{2}\right)\right\} \cup B\right\} \cup \mathcal{C} \in F$, then there is some $S^{\prime} \in \mathcal{F}\left(a_{1} \downarrow a_{2}\right)$ such that $\left\{\{(i, a)\} \cup B \mid a \in S^{\prime}\right\} \cup \mathcal{C} \in F$. Here $S=\left\{a_{1}, a_{2}\right\}$ and $\phi=a_{1} \downarrow a_{2}$. If $\left\{a_{1}\right\} \cup T$ and $\left\{a_{2}\right\} \cup T$ both cover $P_{i}$ then so does $\left(\left\{a_{1}\right\} \cup T\right) \downarrow\left(\left\{a_{2}\right\} \cup T\right)$ and hence so does some finite subset. This enables us to find $S^{\prime}$.

For scheme 3, if $i \in I, a \triangleleft_{0} U$ in $P_{i}, B \in P$ and $\{\{(i, a)\} \cup B\} \cup \mathcal{C} \in F$, then there is some $U_{0} \in \mathcal{F} U$ such that $\left\{\{(i, u)\} \cup B \mid u \in U_{0}\right\} \cup \mathcal{C} \in F$. Here $S=\{a\}$ and $\phi=U$. If $\{a\} \cup T \in F_{i}$ then by hypothesis $U_{0} \cup T \in F_{i}$ for some $U_{0} \in \mathcal{F} U$.

Condition 4, if $\mathcal{A} \in F$ then $P \triangleleft \mathcal{A}$. Let us write

$$
\mathcal{B}^{\prime}=\{\operatorname{Im} \gamma \mid \gamma \in \operatorname{Ch}(\mathcal{A})\} .
$$

For every $\gamma \in \operatorname{Ch}(\mathcal{A})$ we can find $S$ in some $F_{i}$ such that $\{i\} \times S \subseteq \operatorname{Im} \gamma$, and it follows that we can find $\mathcal{B} \in \mathcal{F} P$ such that

- every $B$ in $\mathcal{B}$ is $\{i\} \times S$ for some $i$ and $S \in F_{i}$;

- every $B$ in $\mathcal{B}$ is included in some $\operatorname{Im} \gamma$ in $\mathcal{B}^{\prime}$;

- every $\operatorname{Im} \gamma$ in $\mathcal{B}^{\prime}$ includes some $B$ in $\mathcal{B}$.

The last two imply that $\mathcal{B}^{\prime} \sqsubseteq_{U} \mathcal{B}$ and $\mathcal{B}^{\prime} \sqsubseteq_{L} \mathcal{B}$ (recalling that the order used on $P=\mathcal{F} P^{\prime}$ is $\sqsubseteq_{U}$, which includes $\supseteq$ ).

Now let

$$
\mathcal{C}=\{\operatorname{Im} \delta \mid \delta \in \operatorname{Ch}(\mathcal{B})\} .
$$

We show (i) $\mathcal{C} \sqsubseteq_{L} \mathcal{A}$, and (ii) $(\emptyset) \triangleleft \mathcal{C}$, and these together imply that $(\emptyset) \triangleleft \mathcal{A}$.

For the first, take $\delta \in \operatorname{Ch}(\mathcal{B})$. For every $\gamma \in \operatorname{Ch}(\mathcal{A})$ we have that $\operatorname{Im} \gamma$ includes some $B \in \mathcal{B}$ and so meets $\operatorname{Im} \delta$. By the Diagonalization Lemma of [Vic04b] it follows that $A \subseteq \operatorname{Im} \delta$ for some $A \in \mathcal{A}$. (Classically, if no $A \in \mathcal{A}$ is included in $\operatorname{Im} \delta$ then there is a choice that avoids $\operatorname{Im} \delta$. But with these finite sets there is a constructive proof.)

For the second, we use induction on $\mathcal{B}$. If $\mathcal{B}=\emptyset$, then it has only one choice, which is empty, and so $\mathcal{C}=\{\emptyset\}$. Now suppose it holds for $\mathcal{B}_{0}$; we prove it for $\mathcal{B}=\mathcal{B}_{0} \cup\{\{i\} \times S\}$ where $S \in F_{i}$. We have

$$
\mathcal{C} \supseteq\left\{\operatorname{Im} \delta \cup\{(i, b)\} \mid \delta \in \operatorname{Ch}\left(\mathcal{B}_{0}\right), b \in S\right\} .
$$

By induction,

$$
(\emptyset) \triangleleft\left\{\operatorname{Im} \delta \mid \delta \in \operatorname{Ch}\left(\mathcal{B}_{0}\right)\right\} .
$$


By definition of $\triangleleft_{0}$ for $P$,

$$
\operatorname{Im} \delta \triangleleft_{0}\left\{\operatorname{Im} \delta \cup\{(i, a)\} \mid a \in P_{i}\right\}
$$

Since $S \in F_{i}$, we have $a \triangleleft S$ for each $a \in P_{i}$, and a straightforward induction on the proof of $a \triangleleft S$ then shows that

$$
\operatorname{Im} \delta \cup\{(i, a)\} \triangleleft\{\operatorname{Im} \delta \cup\{(i, b)\} \mid b \in S\}
$$

We can now use transitivity of $\triangleleft$.

Now here is the lemma that was promised in proving condition 3.

Lemma 0.7 Under the hypotheses of Theorem 0.6, suppose we have $i \in I, S \in$ $\mathcal{F} P_{i}$ and $\phi \subseteq P_{i}$ with the property that, for every $T \in \mathcal{F} P_{i}$, if $\forall a \in S .\{a\} \cup T \in F_{i}$ then there is some $S^{\prime} \in \mathcal{F} P_{i}$ with $S^{\prime} \subseteq \phi$ and $S^{\prime} \cup T \in F_{i}$.

Then if $B \in P, \mathcal{C} \in \mathcal{F} P$ and $\{(\{i\} \times S) \cup B\} \cup \mathcal{C} \in F$, there is some $S^{\prime} \in \mathcal{F} P_{i}$ with $S^{\prime} \subseteq \phi$ and $\left\{\{(i, a)\} \cup B \mid a \in S^{\prime}\right\} \cup \mathcal{C} \in F$.

Proof Suppose $B$ and $\mathcal{C}$ are given. Now suppose $\gamma \in \operatorname{Ch}(\mathcal{C})$. For every $a \in S$ we have $\{(i, a)\} \cup \operatorname{Im} \gamma$ covers some $P_{j}$, so there is some $S \in F_{j}$ such that $\{j\} \times S \subseteq\{(i, a)\} \cup \operatorname{Im} \gamma$. We can deduce that either $\operatorname{Im} \gamma$ covers some $P_{j}$, or $\{(i, a)\} \cup \operatorname{Im} \gamma$ covers $P_{i}$. We can therefore decompose $\mathrm{Ch}(\mathcal{C})$ as a union of finite sets, $D \cup D^{\prime}$, such that if $\gamma \in D$ then $\operatorname{Im} \gamma$ covers some $P_{j}$, and if $\gamma \in D^{\prime}$ then $\{(i, a)\} \cup \operatorname{Im} \gamma$ covers $P_{i}$ for every $a \in S$. If $\gamma \in D^{\prime}$ then for each $a \in S$ we can find $T \in P_{i}$ such that $\{a\} \cup T \in F_{i}$ and $\{i\} \times T \subseteq \operatorname{Im} \gamma$, and by taking their union we can assume that a single $T$ does for all the $a$ 's. Then we can find $S^{\prime}$ with $S^{\prime} \subseteq \phi$ and $S^{\prime} \cup T \in F_{i}$. By taking the union of the $S^{\prime}$ s we can assume that a single $S^{\prime}$ such that $\left(\{i\} \times S^{\prime}\right) \cup \operatorname{Im} \gamma$ covers $P_{i}$ for all $\gamma \in D^{\prime}$.

We now show that $\left\{\{(i, a)\} \cup B \mid a \in S^{\prime}\right\} \cup \mathcal{C} \in F$. For any choice of $\{\{(i, a)\} \cup$ $\left.B \mid a \in S^{\prime}\right\} \cup \mathcal{C}$, its image contains a set of the form $\operatorname{Im} \gamma \cup \operatorname{Im} \delta$, where $\gamma \in \operatorname{Ch}(\mathcal{C})$ and $\delta \in \operatorname{Ch}\left(\left\{\{(i, a)\} \cup B \mid a \in S^{\prime}\right\}\right)$. For each $a \in S^{\prime}$, we have either $\operatorname{Im} \delta$ meets $B$ or $(i, a) \in \operatorname{Im} \delta$. If the former holds for some $a$, then there is some choice of $\{(\{i\} \times S) \cup B\} \cup \mathcal{C}$ whose image is a subset of $\operatorname{Im} \gamma \cup \operatorname{Im} \delta$, and from $\{(\{i\} \times S) \cup B\} \cup \mathcal{C} \in F$ we deduce that $\operatorname{Im} \gamma \cup \operatorname{Im} \delta$ covers some $P_{j}$. Alternatively, suppose $\{i\} \times S^{\prime} \subseteq \operatorname{Im} \delta$. It suffices then to know that $\left(\{i\} \times S^{\prime}\right) \cup \operatorname{Im} \gamma$ covers some $P_{j}$. If $\gamma \in D$ then $\operatorname{Im} \gamma$ covers some $P_{j}$, while if $\gamma \in D^{\prime}$ then by construction of $S^{\prime}$ we have $\left(\{i\} \times S^{\prime}\right) \cup \operatorname{Im} \gamma$ covers $P_{i}$. In either case we are done.

\subsection{Synthetic locale theory}

We conclude with some remarks on an approach that promises to lay bare many issues of topology, both point-set and point-free. This is the "synthetic topology" of Escardó [Esc04]. It uses the lambda calculus to express maps, and the Tychonoff theorem (at least, binary Tychonoff) is a good illustration.

Recall that $X$ is compact iff there is a Scott continuous map $\forall$ ! $: \Omega X \rightarrow \Omega$ that is right adjoint to the unique frame homomorphism. For sober spaces, all 
continuous maps are Scott continuous (with respect to the specialization order). Also, the opens of $X$ are equivalent to continuous maps from $X$ to the Sierpinski space $\mathbb{S}$, so we can identify $\Omega X$ with the function space $\mathbb{S}^{X}$. Hence, compactness of $X$ can be expressed by a map $\forall_{X}: \mathbb{S}^{X} \rightarrow \mathbb{S}$ right adjoint to the map $\mathbb{S}^{!}: \mathbb{S} \rightarrow \mathbb{S}^{X}$. We can think of the points of $\mathbb{S}$ as being truth values, the top (open) point $T$ being true, and then $\forall(a)$ is the truth value of " $a=X$ ".

If $X$ and $Y$ are both compact, then the corresponding map for $X \times Y$ can be expressed very easily as

$$
\forall_{X \times Y}(u)=\forall_{Y}\left(\lambda y \cdot \forall_{X}(\lambda x \cdot u(x, y))\right) .
$$

To put it another way, $\forall X \times Y: \mathbb{S}^{X \times Y} \rightarrow \mathbb{S}$ is the composite $\cong ;\left(\forall_{X}\right)^{Y} ; \forall_{Y}$ : $\mathbb{S}^{X \times Y} \cong\left(\mathbb{S}^{X}\right)^{Y} \rightarrow \mathbb{S}^{Y} \rightarrow \mathbb{S}$. This is the required right adjoint, and if everything preserves continuity then we get the required Scott continuity.

But there's an obvious flaw in the argument! The function space $\mathbb{S}^{X}$ only exists if $X$ is locally compact (this holds for locales as well as spaces). Apparently, it proves Tychonoff only for locally compact (and sober) spaces.

However, it is possible to get round this by embedding one's category of spaces in a larger category in which the exponentials exist. Escardó refers to "real" spaces (in the original category) and "complex" spaces (in the supercategory). It then remains only to show that morphisms between the complex function spaces do indeed give the required Scott continuous functions between frames.

For locales, the requisite results have been proved in [VT04]. There the category Loc of locales is embedded (by the Yoneda embedding) in the category Set $^{\mathbf{L o c}^{o p}}$ of presheaves over Loc. The fundamental lemma then is that presheaf morphisms (natural transformations) from $\mathbb{S}^{X}$ to $\mathbb{S}^{Y}$ correspond to Scott continuous functions from $\Omega X$ to $\Omega Y$. This allows us to use the above construction of $\forall X \times Y$ as a proof of binary Tychonoff.

Despite the set-theoretic difficulties, it is to be hoped that a predicative argument can also be found to justify such synthetic methods in formal topology.

The infinitary Tychonoff theorem is less well understood from this point of view, but it seems to play the role of a termination principle for recursive algorithms. 


\section{REFERENCES}

[Coq92] T. Coquand, An intuitionistic proof of Tychonoff's theorem, Journal of Symbolic Logic 57 (1992), 28-32.

[Coq03] T. Coquand, Compact spaces and distributive lattices, Journal of Pure and Applied Algebra 184 (2003), 1-6.

[CSSV03] T. Coquand, G. Sambin, J. Smith, and S. Valentini, Inductively generated formal topologies, Annals of Pure and Applied Logic 124 (2003), 71-106.

[Esc04] Martín Escardó, Synthetic topology of data types and classical spaces, Bellairs Workshop: Domain Theoretic Methods in Probabilistic Processes (P. Panangaden and J. Desharnais, eds.), Electronic Notes in Theoretical Computer Science, vol. 87, Elsevier, 2004.

[FG82] M.P. Fourman and R.J. Grayson, Formal spaces, The L.E.J. Brouwer Centenary Symposium (Troelstra and van Dalen, eds.), North Holland, 1982, pp. 107-122.

[FH79] M.P. Fourman and J.M.E. Hyland, Sheaf models for analysis, Applications of Sheaves (M.P. Fourman, C.J. Mulvey, and D. Scott, eds.), Lecture Notes in Mathematics, no. 753, Springer, 1979, pp. 280-301.

[HM81] Karl H. Hofmann and Michael W. Mislove, Local compactness and continuous lattices, Continuous Lattices: Proceedings, Bremen, 1979 (B. Banaschewski and R.-E. Hoffmann, eds.), Lecture Notes in Mathematics, no. 871, Springer, 1981, pp. 209-248.

[Joh82] P.T. Johnstone, Stone spaces, Cambridge Studies in Advanced Mathematics, no. 3, Cambridge University Press, 1982.

[Joh84] P.T. Johnstone, Open locales and exponentiation, Contemporary Mathematics 30 (1984), 84-116.

[Joh85] P.T. Johnstone, Vietoris locales and localic semi-lattices, Continuous Lattices and their Applications (R.-E. Hoffmann, ed.), Pure and Applied Mathematics, no. 101, Marcel Dekker, 1985, pp. 155-18.

[JT84] A. Joyal and M. Tierney, An extension of the Galois theory of Grothendieck, Memoirs of the American Mathematical Society 309 (1984).

[JV91] P.T. Johnstone and S.J. Vickers, Preframe presentations present, Category Theory - Proceedings, Como 1990 (A. Carboni, M.C. Pedicchio, and G. Rosolini, eds.), Lecture Notes in Mathematics, no. 1488, Springer-Verlag, 1991, pp. 193-212.

[Kel50] J.L. Kelley, The Tychonoff product theorem implies the axiom of choice, Fundamenta Mathematicae 37 (1950), 75-76.

[MLM92] S. Mac Lane and I. Moerdijk, Sheaves in geometry and logic, Springer Verlag, 1992. 
[Neg02] Sara Negri, Continuous domains as formal spaces, Mathematical Structures in Computer Science 12 (2002), 19-52.

[NV97] S. Negri and S Valentini, Tychonoff's theorem in the framework of formal topologies, Journal of Symbolic Logic 62(4) (1997), 1315-1332.

[Sam87] G. Sambin, Intuitionistic formal spaces - a first communication, Mathematical Logic and its Applications (Skordev, ed.), Plenum, 1987, pp. $187-204$.

[Ver86] J.J.C. Vermeulen, Constructive techniques in functional analysis, Ph.D. thesis, University of Sussex, 1986.

[Vic89] S.J. Vickers, Topology via logic, Cambridge University Press, 1989.

[Vic95] S.J. Vickers, Locales are not pointless, Theory and Formal Methods of Computing 1994 (London) (C.L. Hankin, I.C. Mackie, and R. Nagarajan, eds.), Imperial College Press, 1995, pp. 199-216.

[Vic97] S.J. Vickers, Constructive points of powerlocales, Math. Proc. Cam. Phil. Soc. 122 (1997), 207-222.

[Vic99] S.J. Vickers, Topical categories of domains, Mathematical Structures in Computer Science 9 (1999), 569-616.

[Vic02] Steven Vickers, Compactness in locales and formal topology, accepted for Proceedings of 2nd Workshop on Formal Topology, Venice 2002. Draft available on web at http://www.cs.bham.ac.uk/ sjv, 2002.

[Vic04a] S.J. Vickers, The double powerlocale and exponentiation: A case study in geometric reasoning, Theory and Applications of Categories 12 (2004), 372-422.

[Vic04b] Steven Vickers, Entailment systems for stably locally compact locales, Theoretical Computer Science 316 (2004), 259-296.

[VT04] S.J. Vickers and C.F. Townsend, A universal characterization of the double powerlocale, Theoretical Computer Science 316 (2004), 297321 . 